
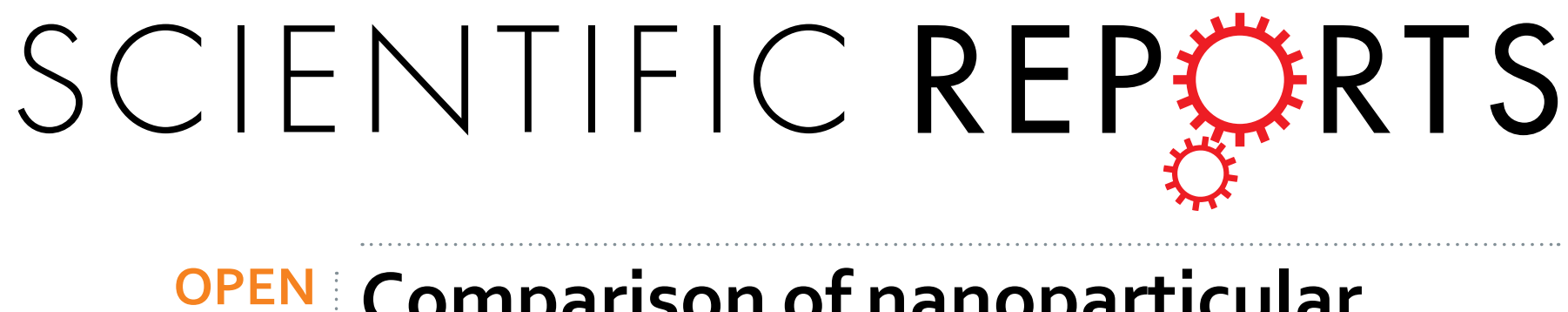

\title{
Comparison of nanoparticular hydroxyapatite pastes of different particle content and size in a novel
}

Received: 13 October 2016 Accepted: 24 January 2017 Published: 24 February 2017

\section{scapula defect model}

Veronika Hruschka ${ }^{1,2}$, Stefan Tang $\left.\right|^{2,3}$, Yulia Ryabenkova ${ }^{4}$, Patrick Heimel ${ }^{1,2,3}$, Dirk Barnewitz ${ }^{5}$, Günter Möbus ${ }^{4}$, Claudia Keib| ${ }^{1,2}$, James Ferguson ${ }^{1,2}{ }^{2}$, Paulo Quadros ${ }^{6}$, Cheryl Miller ${ }^{7}$, Rebecca Goodchild ${ }^{8}$, Wayne Austin ${ }^{8}$, Heinz Redl ${ }^{1,2}$ \& Thomas Nau ${ }^{1,2}$

Nanocrystalline hydroxyapatite (HA) has good biocompatibility and the potential to support bone formation. It represents a promising alternative to autologous bone grafting, which is considered the current gold standard for the treatment of low weight bearing bone defects. The purpose of this study was to compare three bone substitute pastes of different HA content and particle size with autologous bone and empty defects, at two time points ( 6 and 12 months) in an ovine scapula drillhole model using micro-CT, histology and histomorphometry evaluation. The nHA-LC (38\% HA content) paste supported bone formation with a high defect bridging-rate. Compared to nHA-LC, Ostim $®$ ) ( $35 \%$ HA content) showed less and smaller particle agglomerates but also a reduced defect bridging-rate due to its fast degradation The highly concentrated $\mathrm{nHA}-\mathrm{HC}$ paste ( $48 \% \mathrm{HA}$ content) formed oversized particle agglomerates which supported the defect bridging but left little space for bone formation in the defect site. Interestingly, the gold standard treatment of the defect site with autologous bone tissue did not improve bone formation or defect bridging compared to the empty control. We concluded that the material resorption and bone formation was highly impacted by the particle-specific agglomeration behaviour in this study.

Autologous bone graft is considered the gold standard for the treatment of low weight-bearing bone defects. Autologous bone grafts have osteogenic, osteoinductive and osteoconductive properties while being non immunogenic. However, complications at the graft harvesting site, including infection, prolonged wound drainage, large hematomas, vascular injuries, the need for revision surgery, pain, sensory loss, herniation, fracture and cosmetically problematic scars may occur ${ }^{1-7}$. Synthetic substitutes, such as hydroxyapatite (HA) or tricalcium phosphate based materials, also possess osteoconductive properties and present an alternative to autologous bone grafts. Nanocrystalline HA, for example the commercially available paste Ostim ${ }^{\circledR}$, has been successfully used in the fields of oral, maxillofacial, and orthopaedic surgery, showing good biocompatibility and the potential to support bone formation without eliciting an inflammatory response ${ }^{8-14}$. As an injectable paste, Ostim ${ }^{\circledR}$ can be applied in a minimally invasive manner to completely fill irregular defects. Laschke et al. reported that the degradation process of Ostim ${ }^{\circledR}$ enabled cellular migration into the augmentation material and thereby supported vascularisation and facilitated bone formation ${ }^{14}$. Brandt et al. observed agglomerations of the Ostim ${ }^{\circledR}$ nanocrystals that became more dense over time, remaining embedded in bone and connective tissue without further resorption $^{11}$. While single nanoparticles might provoke inflammatory responses and inhibitory effects ${ }^{15,16}$, agglomerates increased the fibronectin adsorption which influences osteoblast adherence ${ }^{17-19}$. The surface topography of HA

${ }^{1}$ Ludwig Boltzmann Institute for Experimental and Clinical Traumatology, AUVA Research Centre, Vienna, Austria. ${ }^{2}$ Austrian Cluster for Tissue Regeneration, Vienna, Austria. ${ }^{3}$ Karl Donath Laboratory for Hard Tissue and Biomaterial Research, Department of Oral Surgery, Medical University of Vienna, Vienna, Austria. ${ }^{4}$ Department of Materials Science and Engineering, The University of Sheffield, Sheffield, United Kingdom. ${ }^{5}$ Research Center for Medical Technology and Biotechnology, Bad Langensalza, Germany. ${ }^{6}$ Fluidinova S.A., Moreira da Maia, Portugal. ${ }^{7}$ The School of Clinical Dentistry, University of Sheffield, Sheffield, United Kingdom. ${ }^{8}$ Ceramisys Ltd., Sheffield, United Kingdom. Correspondence and requests for materials should be addressed to V.H. (email: veronika.hruschka@trauma.Ibg. ac.at) 
plays a critical role in bone regeneration. HA crystals in hard tissues have been reported to be nanoscale rod-like or plate-like crystals ${ }^{20}$. Nanostructured surfaces of implant materials have been shown to enhance cellular activity and induce bone formation ${ }^{14,16,21-24}$. We hypothesize that the HA content and particle size of the nanocrystalline HA paste may affect material behaviour with regard to resorption and bone formation and therefore the in vivo outcome. In our study, three HA pastes differing in HA content and particle sizes were evaluated. Based on preliminary in vitro studies ${ }^{25}$, the following pastes were selected: a paste with a concentration of HA of $38 \%$ and rod shaped particles with a mean length of $40-60 \mathrm{~nm}$ (nHA-LC), a paste with a HA content of $48 \%$ and a similar mean particle length of $40-60 \mathrm{~nm}$ with a more elongated particle shape (nHA-HC) and the commercially available Ostim ${ }^{\circledR}$ with a HA content of $35 \%$, rod shaped particles with reported average dimensions of $150 \mathrm{~nm} \times 25 \mathrm{~nm}^{26}$.

The aim of this study was to compare the potential of two newly developed bone substitute materials to facilitate bone formation, with Ostim ${ }^{\circledR}$, a commercially available product, and the "gold standard" autologous cancellous bone, in an ovine defect model.

\section{Results}

Materials characterisation. X-ray powder diffraction (XRPD) was employed in order to evaluate the phase purity of the test materials. A full match with HA was obtained (full match with the JCPDS card 9-432) with no other phases detected (see Supplementary Fig. 1). The broad peak reflections of the patterns obtained for both nHA-LC and nHA-HC are characteristic of poorly crystalline phases and/or small crystallite size. XRD analysis was not performed on the Ostim ${ }^{\circledR}$ material as the information was readily available from the supplier and stated that it is a phase-pure hydroxyapatite. Thus, we can conclude that both commercially available and in house materials do not contain any other phase and appear to be stoichiometric hydroxyapatites.

Thermogravimetric analysis was performed in order to quantify water content of the non-commercial pastes (see Supplementary Fig. 2). A rapid weight loss up to the temperatures of $c a .400^{\circ} \mathrm{C}$ was observed for both pastes characteristic of the water release from the surface and lattices of hydroxyapatite particles ${ }^{27}$. After this temperature no more weight loss was detected. Thus, it was possible to estimate the amount of water present in the materials at $38 \mathrm{wt} \%$ for $\mathrm{nHA}-\mathrm{LC}$ and $48 \mathrm{wt} \%$ for $\mathrm{nHA}-\mathrm{HC}$, with the former comparable to that of Ostim ${ }^{\circledR}(35 \mathrm{wt} \%)$ and the latter having the highest ceramic loading among all the materials tested. Moreover, no further weight loss was detected after all water had been removed. This shows that materials are thermally stable in the range of temperatures tested, which can be used as further (indirect) evidence of the materials stoichiometry and phase purity ${ }^{28}$.

Transmission electron microscopy was used to visualise the materials constituent particles at the nano-level and estimate their particle size (see Supplementary Fig. 3). Surprisingly, Ostim ${ }^{\circledR}$ did not appear to consist of needle shaped nano-particles of $150 \times 25 \mathrm{~nm}$, as reported earlier in literature ${ }^{14,26}$. On the contrary, it comprised of two different particle types: (a) rod shaped ones with the dimensions of $c a$. $70 \times 20 \mathrm{~nm}$ and (b) interconnected ribbon shaped particles of variable length sometimes exceeding $300 \mathrm{~nm}$. The in-house (non-commercial) materials, comprising mostly of rod shaped nanoparticles with an average length of $c a$. $40-60 \mathrm{~nm}$, slightly varied in the level of roundness, with the ones of nHA-HC being more elongated than that of nHA-LC. This variation may have originated from the preparation method and/or could have been a side effect of the scaling up process of material production.

Interestingly, it was observed that the resulting materials (nHA-LC and nHA-HC) also had different consistencies and appearance: nHA-LC was more paste like, while nHA-HC more like a transparent gel. It was possible to concentrate the nHA-HC produced on a small scale up to nearly $50 \mathrm{wt} \%$ while preserving its injectability properties, whereas nHA-HC, produced at large scale, was hard to concentrate above $c a .40 \%$ as increasing the ceramic content led to a loss of its fluidic properties. It is out of the scope of this manuscript to discuss the differences in the rheological behaviour and optical properties of the pastes and their possible relation to a scale up process, and therefore this manuscript will focus on the in vivo performance of the materials.

Radiography results (x-ray, micro-CT). Animals were sacrificed at 6 months or 12 months after surgery. The $\mathrm{x}$-ray images of the defects filled with the HA pastes Ostim ${ }^{\circledR}$, nHA-LC and nHA-HC indicated that material was present in the defect area at both time points. The defect areas of the control group and of the autologous group were detectable after 6 and 12 months on x-ray images (Fig. 1). The reconstruction of the micro-CT evaluation after 6 months showed a non-bridging or cavity formation in the cortical area of the defects in all groups except the defects filled with nHA-HC (Fig. 2). After 12 months the control group, the autologous group and the defects filled with Ostim ${ }^{\circledR}$ showed a non-bridging or cavity formation at the defect sites, whereas the groups nHA-LC and nHA-HC showed a complete reconstruction of the cortical bone (Fig. 2). nHA-LC, nHA-HC and Ostim ${ }^{\circledR}$ were detectable in the micro-CT at both time points. The pastes, nHA-LC and Ostim ${ }^{\circledR}$ separated into particle units or agglomerates, whereas $\mathrm{nHA}-\mathrm{HC}$ remained mainly compact in the form of a single large agglomerate and showed bone integration only in the periphery of this structure (Fig. 2).

After 6 months, $27 \%$ of the empty control group ( 3 out of 11 ), $17 \%$ of the autologous group ( 2 out of 12 ) and $55 \%$ of the Ostim ${ }^{\circledR}$ filled defects (6 out of 11$)$ presented a bridging, whereas $82 \%$ of the nHA-LC group ( 9 out of 11 ) and $92 \%$ of the nHA-HC filled defects (11 out of 12) showed a bridging of the defect. After 12 months, bridging was detectable for $33 \%$ for the empty control group ( 4 out of 12), 25\% for the autologous group (3 out of 12), $42 \%$ for the Ostim ${ }^{\circledR}$ filled defects ( 5 out of 12 ), but for $75 \%$ of the nHA-LC group ( 9 out of 12 ) and $83 \%$ for the nHA-HC filled defects (10 out of 12).

Histologic evaluation. The margins of the drill holes and their original size were readily detectable in the histologic specimens (see Supplementary Fig. 4). The existing bone present at the edges of the defect stained a lighter shade of pink compared to the new bone tissue that had regenerated inside of the drill hole, which was darker and purple in colour. There were distinct differences between different regions of the defect area. While in the medullary compartment cancellous bone tissue was formed in an amount and in a quality that was 


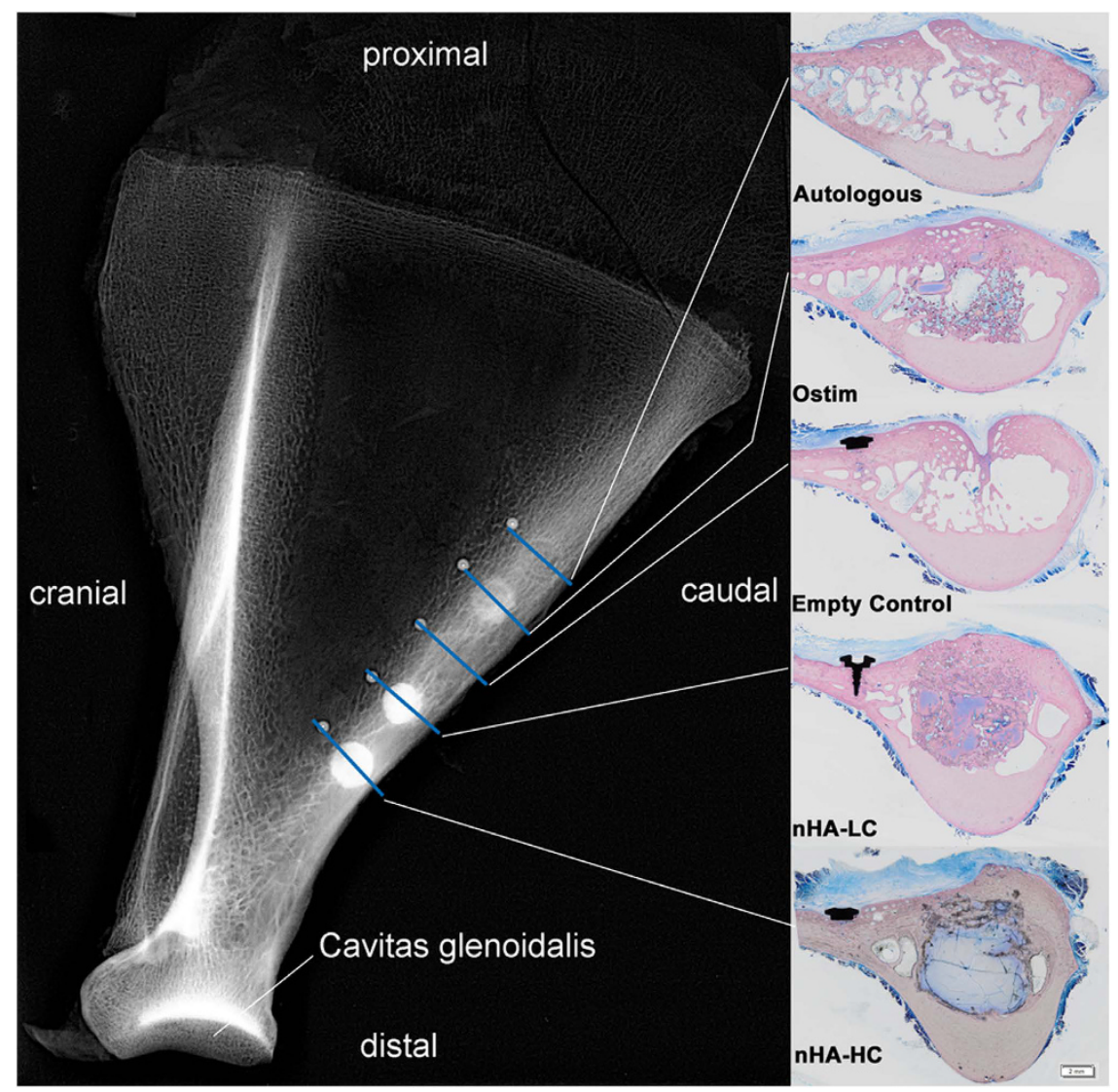

Figure 1. Representative example of the surgical site with marks for the histological sectioning (X-ray, $60 \mathrm{kV}, 0.32 \mathrm{~s}, 6$ months after augmentation): Five defects were placed at the caudal edge of the left scapula and filled in a randomised order with the 4 test substances while 1 was left empty. Every defect site was marked with a titanium pin. Images of the corresponding histological sections, stained with Levai-Laczko dye, are depicted on the right.

comparable to the pre-surgical state, in the cortical area bone regeneration was usually impaired. In the control group which received no bone substitute material at all, deep concave depressions were visible in the cortical bone. Additionally, in many cases the bony cortex was not completely continuous but was breached by canals that connected the bone marrow with the surrounding periosteal region, i.e. the defects were not completely bridged. This indicates that in the chosen animal model no final defect healing is achieved spontaneously, neither after 6 nor after 12 month. Even when treated with autologous bone, depressions, canals and gaps were present in the cortical layer. Only very small remnants of the bone graft were still detectable in the augmented regions.

The three tested HA-pastes exhibited very different results in several aspects. The most obvious was the amount, size and size variation of the remaining agglomerates of HA-paste. In the Ostim ${ }^{\circledR}$ group many of these agglomerations formed numerous particles that had about the size of an osteon $(200 \mu \mathrm{m})$. In the nHA-LC group most of the agglomeration particles were of about the same size, but there were also a few much larger particles, often more than $5 \mathrm{~mm}$ in diameter. This trend was even more pronounced in the nHA-HC group where single particle agglomerations, significantly larger than in the nHA-LC group, were detectable, often filling the whole defect.

Concerning the regeneration of the cortical layer, Ostim ${ }^{\circledR}$ and nHA-LC exhibited results that were comparable to the control groups. The cortical areas showed depressions and canals connecting the marrow space with the soft tissue surrounding the scapula although not as severe and frequent as in the controls. In the nHA-HC group on the other hand the cortical bone overlaying the large agglomerations was more continuous, depressions less pronounced or absent and the prevalence of holes in the compact bone lower.

No great differences were seen in the osteoconductive properties of the bone substitutes. Most of the surfaces of all three materials were covered with thin layers of vital, newly formed bone, demonstrating their ability to enhance and promote bone regeneration. On those parts of the surfaces not covered with bone, very often signs of resorption could be seen. These occurred in the form of resorbing, multinucleated cells, resorption lacunae and more often discoloured seams that are characteristic of the cellular degradation of bone substitute materials.

Histological findings changed comparatively little between 6 and 12 months. Most prominent is the reduction of the size of particle agglomerations also indicating that resorption of HA-pastes is continuously proceeding over considerable periods of time. 

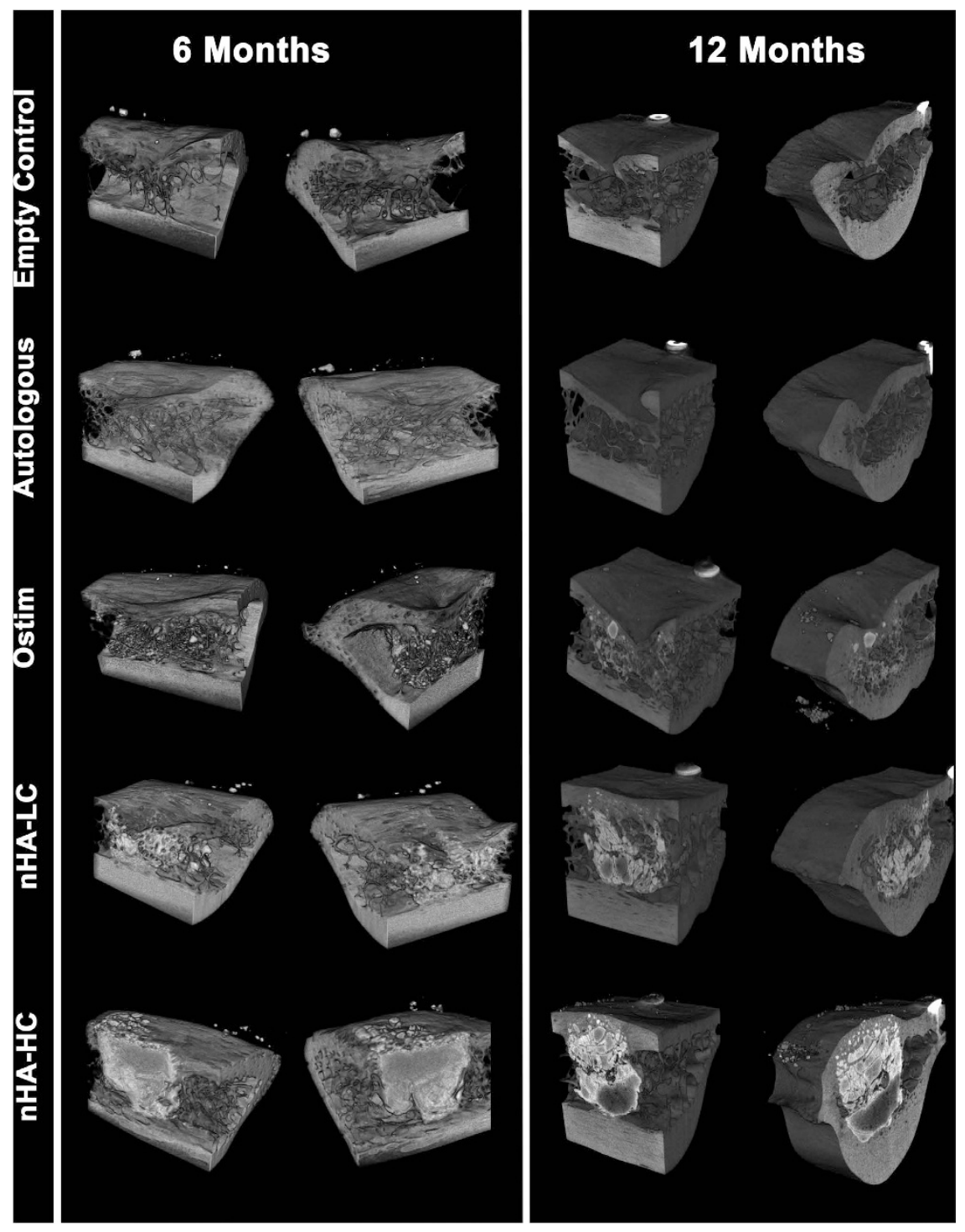

Figure 2. Micro-CT reconstructions of representative defect sites 6 months (left) and 12 months (right) after implantation. Defects left empty or filled with autologous bone or Ostim ${ }^{\circledR}$ showed a non-bridging or cavity formation in the cortical region. $\mathrm{nHA}-\mathrm{LC}$ and $\mathrm{nHA}-\mathrm{HC}$ were able to bridge the defect site at the cortical area. A high amount of dense nHA-HC was found in the defect site.

Histomorphometric evaluation. Bone Substitute Volume/Tissue Volume. The BS.V/TV describes the percentage of bone substitute material in the cortical or medullary defect areas. Compared to Ostim ${ }^{\circledR}$, a statistically significant increase in BS.V/TV was detected at 6 months in both regions of interest for nHA-LC $(\mathrm{p}<0.001)$ as well as nHA-HC $(\mathrm{p}<0.001)$. At 12 months, the BS.V/TV was increased in the medullary region for nHA-LC $(\mathrm{p}<0.001)$ and nHA-HC $(\mathrm{p}<0.001)$ compared to Ostim ${ }^{\circledR}$. In the cortical region at 12 months elevated levels of BS.V/TV of defects filled with nHA-HC were detected compared to Ostim ${ }^{\circledR}(\mathrm{p}<0.001)$ and nHA-LC $(\mathrm{p}<0.001)$ (Fig. 3).

Number and size of bone substitute particles. The nanoparticles of the three different pastes formed larger particle agglomerates, their size and number could then be determined on the histological sections. In the cortical region, nHA-LC showed the highest BS. Pa.N. per $\mathrm{mm}^{2}$ compared to Ostim ${ }^{\circledR}$ ( $\mathrm{p}<0.001$ at 6 months, $\mathrm{p}<0.015$ at 12 months) and nHA-HC ( $\mathrm{p}<0.001$ at 6 and 12 months). In the medullary region at 6 months, a significant decrease of nHA-HC BS.Pa.N/TV was found compared to Ostim ${ }^{\circledR}(\mathrm{p}<0.033)$ and nHA-LC $(\mathrm{p}<0.003)$. At 12 months, a decreased amount of nHA-HC BS.Pa.N/TV was measured compared to nHA-LC (p0.001) (Fig. 4a).

The BS.Pa.Ar. of all pastes showed a tendency to decrease from month 6 to month 12, indicating a certain break-down and resorption of the material (Fig. 4b). At 6 months, no significant difference in BS.Pa.Ar. could be defected in the cortical region. In the medullary region an increased nHA-HC particle agglomerate size was detected compared to Ostim ${ }^{\circledR}(\mathrm{p}<0.001)$ and nHA-LC $(\mathrm{p}<0.001)$. At 12 months, the BS.Pa.Ar. of nHA-HC in 


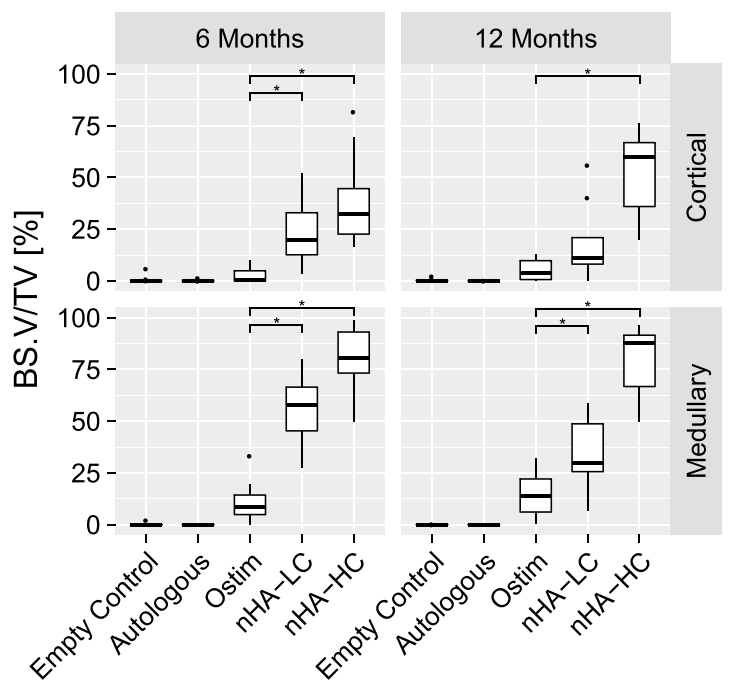

Figure 3. Bone substitute volume (BS.V.) per tissue volume (TV): percentage of the regions of interest that are filled with bone substitute material. Among the three HA-paste fillings, nHA-HC showed the highest and Ostim ${ }^{\circledR}$ the lowest BS.V/TV ratios at both time points in both areas of interest $\left({ }^{*} \mathrm{p}\right.$-value $<0.05$ compared to Ostim $\left.^{\circledR}\right)$.
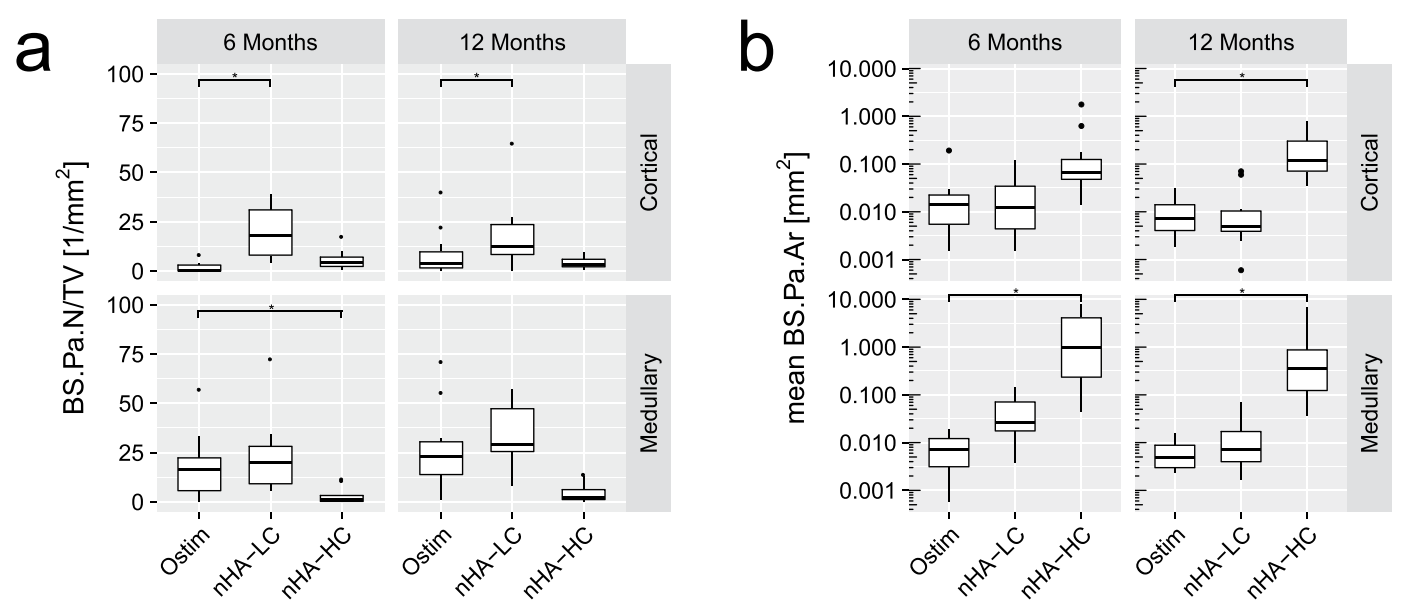

Figure 4. (a) Number of bone substitute particle agglomerates (BS.Pa.N) per tissue volume (TV): In the cortical region, an increase in nHA-LC BS.Pa.N/TV was detected. In the medullary region at 6 months, a decrease in nHA-HC BS.Pa.N/TV was measured. (b) Average size of bone substitute particle agglomerates (BS.Pa.Ar) as measured in the region of interest in $\mathrm{mm}^{2}$ presented on a logarithmic scale. nHA-HC showed an increased agglomerate size with means ranging from 0.22 to $2.67 \mathrm{~mm}^{2}$, compared to the mean agglomerate sizes of nHA-LC and Ostim ${ }^{\circledR}$ ranging below $0.051 \mathrm{~mm}^{2}\left({ }^{\star}\right.$ p-value $<0.05$ compared to Ostim $\left.{ }^{\circledR}\right)$.

the cortical and medullary region was found to be significantly increased compared to Ostim ${ }^{\circledR}$ ( $\mathrm{p}<0.001$ and $\mathrm{p}=0.026$, respectively) and nHA-LC $(\mathrm{p}<0.001$ and $\mathrm{p}=0.028$, respectively) (Fig. $4 \mathrm{~b}$ ).

Newly formed bone volume and the composite volume of bone substitute and newly formed bone in the regions of interest. At 6 months, no significant differences in $\mathrm{nBV} / \mathrm{TV}$ were measured in the cortical region. In the medullary region a reduced amount of $\mathrm{nBV} / \mathrm{TV}$ was detected for the defects filled with nHA-HC compared to the empty control $(p=0.018)$, the autologous bone $(p=0.038)$ and $\operatorname{Ostim}^{\circledR}(p<0.001)$. Also between the defects treated with Ostim ${ }^{\circledR}$ and nHA-LC a significant difference was found $(\mathrm{p}=0.017)$ (Fig. 5a). At 12 months, a significant decrease in $\mathrm{nBV} / \mathrm{TV}$ in the cortical region between defects augmented with nHA-LC and nHA-HC was detected $(\mathrm{p}=0.011)$. In the medullary region defects filled with $\mathrm{nHA}-\mathrm{HC}$ showed a decrease in $\mathrm{nBV} / \mathrm{TV}$ compared to all other groups $(\mathrm{p}<0.001)$ (Fig. 5a).

The highest amount of composite volume, which is the sum of volumes of newly formed bone and bone substitute material, per TV was found at both time points and in both areas of interest in the defects filled with nHA-HC. Up to $97.1 \%$ (12 months, cortical region) of the region of interest treated with nHA-HC consisted of composite material (Fig. 5b). Defects filled with nHA-LC showed Co.V/ TV means in the cortical region of $82.1 \%$ (month 6 ) 

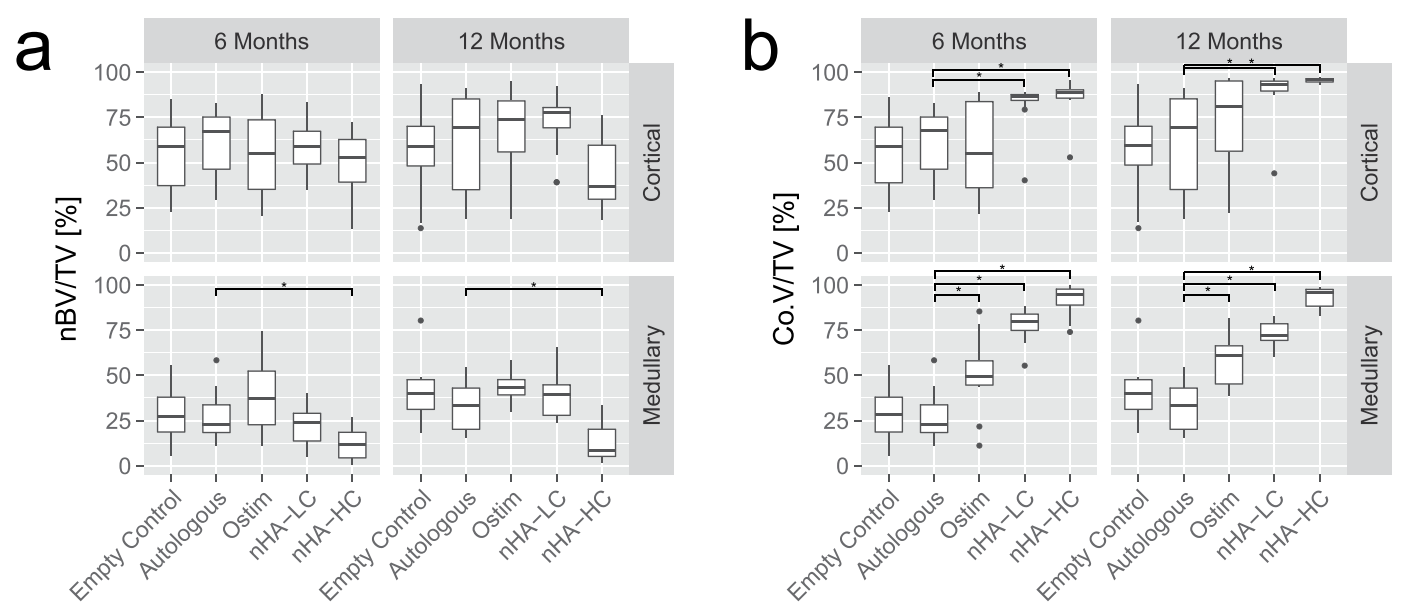

Figure 5. (a) Newly formed bone volume (nBV) per tissue volume (TV): Comparable results were found between the defect fillings at both regions of interest and both time points, except the defects filled with nHA-HC, which showed a lower bone bone volume per TV in the medullary region. (b) Defects filled with Ostim $^{\circledR}$, nHA-LC and nHA-HC showed an increased composite volume (Co.V) per tissue volume (TV) compared to the autologous group at both time points in the medullary region $(\mathrm{p}<0.001)$. In the cortical region, a significant increase at both time points in Co.V/TV between defects filled with nHA-LC and nHA-HC and the autologous group $(\mathrm{p} \leq 0.018)$ were found $\left({ }^{\star} \mathrm{p}\right.$-value $<0.05$ compared to autologous control).

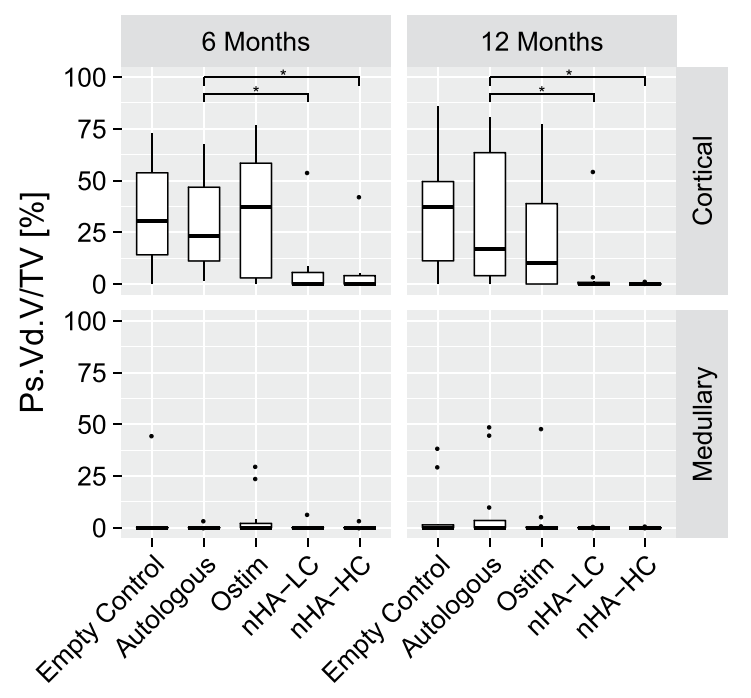

Figure 6. Periosteal void volume (Ps.Vd.V) per tissue volume (TV) presenting the soft tissue ingrowth into the defect site. Very limited soft tissue ingrowth was detected in the medullar region for all treatment groups and at both time points. In contrast, soft tissue ingrowth was found in the cortical region when the defects were filled with Ostim ${ }^{\circledR}$, autologous bone or left empty. nHA-HC and nHA-LC showed a decreased soft tissue ingrowth at both time points ( ${ }^{*} \mathrm{p}$-value $<0.05$ compared to autologous control).

and $88.8 \%$ (month 12 ); and in the medullary region of $77.6 \%$ (month 6 ) and $73.1 \%$ (month 12). Defects augmented with Ostim ${ }^{\circledR}$ resulted in Co.V/TV means in the cortical region of $56.8 \%$ (month 6) and $71.4 \%$ (month 12) and in the medullary region of $49.9 \%$ (month 6) and 58.6\% (month 12) (Fig. 5b).

Soft tissue ingrowth-Periosteal void volume per TV. The PS.Vd.V/TV shows the percentage of soft tissue that has penetrated from the periosteal region into the defect area. In general, more soft tissue ingrowth was found in the cortical region compared to the medullary region. No significant difference was found in the medullary region between the groups at both time points. In the cortical region at 6 months, a significant decrease of soft tissue ingrowth was found for the defects filled with nHA-LC compared to the empty control group $(\mathrm{p}=0.004)$, the autologous group $(\mathrm{p}=0.034)$ and $\mathrm{Ostim}^{\circledR}(\mathrm{p}=0.006)$. Similarly, the defects augmented with nHA-HC showed a significant decrease in soft tissue ingrowth compared to the empty control $(\mathrm{p}=0.002)$, the autologous group $(p=0.019)$ and Ostim ${ }^{\circledR}(p=0.003)$. Also after 12 months, less soft tissue ingrowth was observed for defects filled with nHA-LC compared to the empty control $(p=0.001)$ and the autologous group $(p=0.010)$. Defects filled with nHA-HC showed a decreased soft tissue ingrowth compared to the empty control $(p<0.001)$, the autologous 
group $(\mathrm{p}=0.001)$ and Ostim ${ }^{\circledR}(\mathrm{p}=0.040)$ (Fig. 6). No significant differences were detected between Ostim ${ }^{\circledR}$ and the control groups or between nHA-LC and nHA-HC.

A summary of the histomorphometric evaluation can be found in Supplementary Table 1 (cortical region) and Supplementary Table 2 (medullary region).

\section{Discussion}

It has been reported that sheep present a valuable model for human bone turnover and remodelling. It was demonstrated that the ovine bone ingrowth and mineral acquisition into porous-coated implants was comparable to that of humans ${ }^{29}$. Additionally, the ovine weight and bone dimensions are more similar to humans than other animal models and furthermore, aged sheep have been also shown as suitable model for human osteopenic and osteoporotic bone $\mathrm{e}^{30,31}$. While the $\mathrm{x}$-ray evaluation of the bones confirmed that the defects were placed at the same locations of the left scapula, the micro-CT showed that only the upper cortical bone and medullary regions were involved, whereas the lower cortical region remained intact. The empty defects resulted in non-bridging (average of $70 \%$ of the defects) or cavity formation, suggesting that an adequate model and time points were chosen.

$\mathrm{HA}$ is widely used in the clinics and has been reported as a biocompatible and osteoconductive bone substitute material ${ }^{8,9,11,14,32,33}$. Ostim ${ }^{\circledR}$, a nanoparticular HA aqueous paste, has been described as bone-inducible paste. Several publications observed that Ostim ${ }^{\circledR}$ particles were not fully resorbed 12 weeks post-surgery $y^{9,11,12,34}$. In general all HA pastes used in this study were shown to support bone formation. 12 months post-surgery the three HA pastes were, to varying degrees, detectable in all defects. The HA content of the pastes related to the amount of BS.V/TV, and Co.V/TV. However, as the particle size and shape also varies among the three pastes, the performance of the pastes cannot be merely attributed to their HA content. We observed nanoparticle agglomerates of various sizes for all three pastes. nHA-HC agglomerates presented mean sizes ranging from $0.22 \mathrm{~mm}^{2}$ to $2.67 \mathrm{~mm}^{2}$. In the medullary region, nHA-LC agglomerates showed mean sizes of $0.051 \mathrm{~mm}^{2}$ after 6 months and $0.017 \mathrm{~mm}^{2}$ after 12 months. The mean agglomerate size of Ostim ${ }^{\circledR}$ in the medullary region was $0.008 \mathrm{~mm}^{2}$ after 6 months and $0.006 \mathrm{~mm}^{2}$ after 12 months. These differences in agglomeration behaviour may explain the significantly quicker resorption of Ostim ${ }^{\circledR}$ compared to nHA-LC that has a similar HA content. The BS.Pa.N/TV evaluation underlined the particle aggregation of the nHA-HC paste resulting in a very low particle number but large particle size. The trend of BS.Pa.N/TV increase along with the tendency of a mean BS.Pa.Ar. decrease for nHA-LC and Ostim ${ }^{\circledR}$ in the medullary region from month 6 to 12 indicated the break-down of the particle agglomerates into smaller agglomerate subunits. The defects filled with Ostim ${ }^{\circledR}$ and autologous bone resulted in a cavity formation and a low ratio of bridging defects (average of $48 \%$ for Ostim ${ }^{\circledR}$ and $21 \%$ for autologous bone). In contrast, defects filled with nHA-LC and nHA-HC showed a cortical bone formation with an average bridging ratio of $78 \%$ (nHA-LC) and $88 \%$ (nHA-HC), respectively. This effect was observed in the micro-CT evaluation and confirmed by the Ps.Vd.V/TV results, showing soft tissue ingrowth in the defect sites. Dau et al. created mono cortical critical size bone defects in minipigs that were filled with the two nanoparticular HA pastes Ostim ${ }^{\circledR}$, NanoBone ${ }^{\circledR}$ or the natural bone substitute material Bio-Oss ${ }^{\circledR}$, which is derived from the mineral portion of bovine bone. While after 8 months, Ostim ${ }^{\circledR}$ showed the least amount of residual material at the defect sites, no differences in new bone formation or soft tissue ingrowth was detected between the tested groups. They recommended NanoBone ${ }^{\circledR}$ or Bio-Oss ${ }^{\circledR}$ for use in defects that require stability of the bone ${ }^{35}$. In contrast to Dau et al., our study detected an increased soft tissue ingrowth in the Ostim ${ }^{\circledR}$ group compared to the other HA pastes. Based on our findings nHA-LC should be chosen for stable bone integration.

Interestingly, autologous bone grafts failed to improve bone formation or decrease soft tissue ingrowth, giving comparable results to the empty control. Bone grafts undergo extensive remodelling and resorption during the first year. Dependent on the nature of the grafted material and implantation site, various autologous bone resorption rates have been reported ${ }^{36}$. A $29 \%$ resorption rate of autologous bone grafts from the iliac crests used for sinus floor augmentation was observed after 3 months ${ }^{37}$. Van der Meij et al. reported the presence of approximately $30 \%$ of autologous bone graft from the iliac crest for cleft lip and palate treatment after 1 year ${ }^{38}$. Autologous bone blocks for horizontal ridge augmentation lost $28 \%$ of its volume during 6 months ${ }^{39}$. In our study a low amount of the autologous cancellous bone was detectable at both time points, indicating a fast resorption of the grafted material. The fast resorption of the autologous bone graft may explain the similar results to the empty defects. Several publications reported a size-dependent cytotoxic effect of nanoparticles in vitro and in vivo when administering particle suspensions $s^{40-43}$. Clinical studies evaluating the suitability of Ostim ${ }^{\circledR}$ as a bone-filling matrix reported good bone induction with limited or no side effects ${ }^{44-48}$. Huber et al. reported that biopsy analysis showed good bone formation without inflammatory reactions, osteofibrosis or osteonecrosis ${ }^{48}$. In this study we observed good bone regeneration for all three HA pastes without any inflammatory reaction, osteofibrosis or osteonecrosis. The liquid phase of the water-based HA pastes can decrease over time, which could explain the formation of dense particle agglomerates ${ }^{11}$. Therefore, the previous studies evaluating monodisperse particle solution might not represent the observed in vivo paste behaviour.

In this study we observed a strong correlation between HA content, the size of the particle agglomerates with the amount of newly formed bone tissue and defect bridging. While a high HA content of $48 \mathrm{wt} \%$ with larger particle agglomerates supported defect bridging, less newly formed bone tissue was obtained within the defect site. This is probably caused by the fact that large particle agglomerates leave less space for the formation of new bone. On the other side, a lower HA content of $35 \mathrm{wt} \%\left(\mathrm{Ostim}^{\circledR}\right)$ and $38 \mathrm{wt} \%$ (nHA-LC), respectively, was correlated with smaller particle agglomerates, an increased amount of newly formed bone tissue within the defect but also a reduced defect bridging ratio. The impact of particle agglomeration behaviour must be examined in more detail in further studies. 


\section{Conclusion}

In this preclinical study, three bone graft pastes of different HA concentrations and particle sizes were compared to treatment of defects with autologous bone and empty defects. Bone regeneration and growth but incomplete defect healing was observed in the empty control group and by treatment with autologous bone. However, a cavity at the defect site was visible. While the higher concentrated (48 wt $\%)$ nHA-HC agglomerated and supported bone formation only on the outer surface of the material, Ostim ${ }^{\circledR}(35 \mathrm{wt} \%)$ degraded the quickest and could not fill the defect for a sufficient period of time to allow for a complete cortical bone bridging. This was similar to the autologous bone filling where a cavity at the defect site, an increase in soft tissue ingrowth and a low bridging rate were observed. We suggest that the agglomeration behaviour presents a critical parameter for nanoparticular HA pastes due to their influence on material resorption and bone formation. nHA-LC (38 wt\%) seemed to have the best combination of characteristics that lead to good bone regeneration, reduced soft tissue ingrowth, had a high defect bridging rate and optimal particle agglomeration size, while keeping in mind the limitations of animal studies.

\section{Materials and Methods}

Materials preparation and characterisation. Commercially available Ostim ${ }^{\circledR}$ was obtained from Heraeus Kulzer, Germany. Nano-hydroxyapatite (nHA) was prepared as previously described ${ }^{49}$. In brief, aqueous solutions of calcium chloride and potassium phosphate monohydrate were fed into the inlets of either large or small scale NETmix reactors at a molar ratio of 1.67, or 5:3 $3^{50}$, under controlled $\mathrm{pH}$ of 11-12 using potassium hydroxide (all reagents from Sigma-Aldrich, Spain). Resultant slurries were further concentrated to viscous pastes/gels of $c a .38$ and $48 \mathrm{wt} \%$ and were given codes nHA-LC for material prepared in a large scale reactor and nHA-HC for material from a small scale reactor, respectively.

Thermogravimetric analysis (TGA) was performed on nHA-LC and nHA-HC materials to quantify the concentration of water and ceramics (nHA) in the systems. TGA was carried out using a PerkinElmer Pyris 1 thermogravimetric analyser. Heating was performed in a platinum crucible in nitrogen flow $\left(20 \mathrm{~cm}^{3} \cdot \mathrm{min}^{-1}\right)$ at a rate of $10^{\circ} \mathrm{C} \mathrm{min}{ }^{-1}$ from $30^{\circ} \mathrm{C}$ up to $1000^{\circ} \mathrm{C}$. Proportions of the materials were dried at $60^{\circ} \mathrm{C}$ and further ground for performing phase analysis using $\mathrm{x}$-ray powder diffraction (XRPD) method.

Aliquots of the pastes as well as Ostim ${ }^{\circledR}$ were dissolved in distilled water and dispersed onto a holey carbon film supported by a 300 mesh copper grid for particle size evaluation using a transmission electron microscope (TEM) JEOL JEM-3010 with a $\mathrm{LaB}_{6}$ electron gun at an accelerating voltage of $300 \mathrm{kV}$.

$\mathrm{X}$-ray powder diffraction (XRPD) patterns were acquired using a Siemens D5000 Diffractometer operating at $40 \mathrm{kV}$ and $40 \mathrm{~mA}$ with $\mathrm{Cu} \mathrm{K \alpha}$ radiation. Analysis of the obtained patterns was carried out using X'Pert HighScore Plus software, and stripping of the $\mathrm{K} \alpha-2$ component was carried out when appropriate.

Ethical conduct of research. The study was approved by the local Thuringia State Office ethical committee (“Thüringer Landesamt für Verbraucherschutz") and was conducted in accordance with the principles of the 1964 Declaration of Helsinki.

Animals and anaesthesia. For this study, 24 adult (age of 2-3 years) female land merino sheep that during their life had given birth at least 1-2 times were housed in a freestall barn, fed with hay and allowed to drink water ad libitum. The study was approved by the local ethical committee for animal experiments (see chapter Ethical conduct of research). Haematological and parasitological examinations were performed prior to the study. Animals were operated in two groups in the late autumn. General anaesthesia was induced by an intravenous administration of $0.1 \mathrm{mg} / \mathrm{kg}$ Diazepam, $4.4 \mathrm{mg} / \mathrm{kg}$ Ketamin and $0.1 \mathrm{mg} / \mathrm{kg}$ Xylazin. After induction, the animals were intubated and connected to a semi-open anaesthesia system with an isoflurane concentration of 1.0-1.5 volume $\%$ and an oxygen supply of $21 \mathrm{O}_{2} / \mathrm{min}$. The animals received a pre-operative systemic antibiotic $(3 \mathrm{ml} / 50 \mathrm{~kg}$ Veracin-Compositum i.m.) as well as the analgeticum Rimadyl ${ }^{\circledR}$ (Pfizer, $1 \mathrm{ml} / 35 \mathrm{~kg}$ i.v.).

Surgical procedure, material application and post-operative medication. A longitudinal incision along the caudal border of the left scapula was made and the border was exposed by sharp dissection. Using a trephine drill, five defects with a diameter and depth of $8 \mathrm{~mm}$ were created, leaving a $5 \mathrm{~mm}$ bony bridge in between each defect ${ }^{51}$. The longitudinal axis of the drill holes was oriented perpendicular to the lateral face of the scapula. The bony cylinders from the drill holes were preserved for autologous bone grafting. Titanium pins were placed close to the periphery of the holes to mark the exact location of the defect areas. One defect remained empty and served as a control. The other defects were filled with one of four materials: (1) autologous bone graft, (2) the commercially available Ostim ${ }^{\circledR}$ with a HA content of $35 \mathrm{wt} \%$, (3) injectable paste with a concentration of $38 \mathrm{wt} \%$ HA-crystals (nHA-LC) and (4) paste with a high concentration of $48 \mathrm{wt} \%$ HA-crystals (nHA-HC) (Fig. 1). The five groups were randomised to the defect sites for each animal prior to the procedures. The surgical site was closed in multiple layers using Monocryl 3-0 suture material. The animals received Rimadyl ${ }^{\circledR} 1 \mathrm{ml} / 35 \mathrm{~kg}$ s.c. five days post-operatively. Twelve animals were euthanised after either 6 or 12 months by the administration of T $61^{\circledR}$ i.v.

Histology and sample preparation. The scapulae were removed and fixed in buffered formalin. Overview radiographic images were produced to ascertain the exact location of the augmentation sites. Blocks, each of them containing one of the defects were dissected using a diamond coated band saw (EXAKT Apparatebau, Norderstedt, Germany) by cutting precisely in between the drill holes as visible on the radiograph. These blocks were subjected to micro-CT ( $\mu \mathrm{CT}$ ) analysis (see section "Micro-CT analysis"). After the $\mu \mathrm{CT}$ scan the specimen blocks were dehydrated, embedded in plastic and then undecalcified thin sections were produced using the method of Donath ${ }^{52}$. Histologic specimens were stained with Levai-Laczko dye and photographed with an 
Olympus BX61VS scanning microscope (Olympus DotSlide system 2.4; Olympus, Tokyo, Japan) at a resolution of $0.321 \mu \mathrm{m} /$ pixel.

Micro-CT analysis. The samples were scanned in a SCANCO $\mu$ CT 50 at $90 \mathrm{kV}$ with an isotropic resolution of $17.2 \mu \mathrm{m}$. The scans were exported as 16 bit DICOM image stacks. Based on the micro-CT evaluation the closure of the defects was categorised as "non-bridging" (leaving a continuous opening in the cortical bone connecting the medullary cavity with the periosteal area) or "bridging" when the continuity of the cortical bone was completely restored and no opening remained.

Histomorphometric analyses. The histological images were downsampled to $25 \%(1.283 \mu \mathrm{m} / \mathrm{pxl})$ and then automatically segmented using Definiens Developer XD 2.1 (Definiens AG, Munich, Germany). Errors in segmented images were corrected manually using Photoshop CS 4 (Adobe Systems Inc., San Jose, CA, USA). The corrected images were then measured in Definiens Developer XD 2.1. A quadtree segmentation, in which square objects are quartered until they fulfil set homogeneity criteria, was applied to the images. The resulting square objects were first classified in a very rough manner to determine the colour balance of the individual image. The background (soft tissue) was classified using a very conservative threshold and then grown into surrounding background using a less restrictive threshold. Bone in the remaining area was classified using differences in the colour layers. Bone objects which were not bordered by other bone objects on at least two sides were declassified to remove potential misclassified bone objects. In the resulting segmented bone, the mean difference between the red and green layers were measured and used to calculate the threshold for a more accurate segmentation. Bone and the unclassified area around it was thresholded using this calculated threshold on the pixel level. The border between bone and background was smoothed using alternating pixel based growing and shrinking constrained by surface tension. Small objects completely surrounded by another class were added to the surrounding class. The border was further improved by allowing it to shift position slightly if this increased the difference between the separated classes in the area. The segmentation was exported for manual correction. The regions of interest were selected before the correction. The cortical region of interest was defined as the area between the boundaries of the drill hole in the cortical bone and the periosteal and endosteal margins. The medullary region of interest was located $1 \mathrm{~mm}$ beneath the cortical region of interest, was oriented parallel to it and had a height of $1 \mathrm{~mm}$. The medullary region of interest also spanned the whole breadth of the drill hole and lay solidly within the marrow space.

As the substitute materials could not be reliably distinguished automatically these were classified entirely manually. The corrected images were then measured in Definiens. The areas of newly formed bone (nBV), background or soft tissue (Vd.V), substitute material (BS.V) and the complete region of interest (TV; tissue volume) were measured. Also the sizes of the individual particle agglomerates (BS.Pa.V) and their number (BS.Pa.N) were determined. From these primary measurements the following parameters were calculated: The percentage of bone substitute material in the regions of interest (BS.V/TV), the average size of particle agglomerates (BS.Pa.Ar) in $\mathrm{mm}^{2}$, the number of bone substitute particle agglomerates per $\mathrm{mm}^{2}$ (BS.Pa.N/TV), the percentage of newly formed bone in the regions of interest $(\mathrm{nBV} / \mathrm{TV})$, the percentage of the composite of newly formed bone plus bone substitute material (Co.V/TV), the percentage of the region of interest filled with soft tissue that had grown from the periosteal region into the defect area (Ps.Vd.V; Periosteal void volume).

Statistical analysis. All animals were included in the final analysis; one defect site $\left(6\right.$ months, Ostim $\left.{ }^{\circledR}\right)$ was excluded due to faulty drilling. Two other defects (6 months, empty control and autologous) were excluded from the bridging/non-bridging analysis due to later micro-CT reconstruction problems. For inference, multiple linear mixed models for all independent variables were built using treatment as independent variable and animal ID as a random factor. We then proceeded to calculate Tukey-type post-hoc tests for treatment effect $t^{53}$. All computations were done using R version 3.2.3 $3^{54}$. Statistical Graphs were created using ggplot $2^{55}$.

\section{References}

1. Younger, E. M. \& Chapman, M. W. Morbidity at bone graft donor sites. J. Orthop. Trauma 3, 192-195 (1989).

2. Ahlmann, E., Patzakis, M., Roidis, N., Shepherd, L. \& Holtom, P. Comparison of anterior and posterior iliac crest bone grafts in terms of harvest-site morbidity and functional outcomes. J. Bone Joint Surg. Am. 84-A, 716-720 (2002).

3. Calori, G. M. et al. Incidence of donor site morbidity following harvesting from iliac crest or RIA graft. Injury 45 Suppl 6, S116-120 (2014).

4. Kim, D. H. et al. Prospective study of iliac crest bone graft harvest site pain and morbidity. Spine J9, 886-892 (2009).

5. Banwart, J. C., Asher, M. A. \& Hassanein, R. S. Iliac crest bone graft harvest donor site morbidity. A statistical evaluation. Spine 20, 1055-1060 (1995).

6. Arrington, E. D., Smith, W. J., Chambers, H. G., Bucknell, A. L. \& Davino, N. A. Complications of iliac crest bone graft harvesting. Clin. Orthop. 300-309 (1996).

7. Pape, H. C., Evans, A. \& Kobbe, P. Autologous bone graft: properties and techniques. J. Orthop. Trauma 24 Suppl 1, S36-40 (2010).

8. Arabmotlagh, M., Sommer, U., Dingeldein, E., Rauschmann, M. \& Schnettler, R. Nanocrystalline hydroxyapatite facilitates bone apposition to polymethylmethacrylate: Histological investigation using a sheep model. J. Orthop. Res. 30, 1290-1295 (2012).

9. Busenlechner, D. et al. Simultaneous in vivo comparison of bone substitutes in a guided bone regeneration model. Biomaterials 29, 3195-3200 (2008).

10. Müller-Mai, C. M., Stupp, S. I., Voigt, C. \& Gross, U. Nanoapatite and organoapatite implants in bone: histology and ultrastructure of the interface. J. Biomed. Mater. Res. 29, 9-18 (1995).

11. Brandt, J. et al. Nanocrystalline hydroxyapatite for bone repair: an animal study. J. Mater. Sci. Mater. Med. 21, 283-294 (2009).

12. Huber, F.-X. et al. Evaluation of a novel nanocrystalline hydroxyapatite paste Ostim ${ }^{\circledR}$ in comparison to Alpha-BSM ${ }^{\circledR}-$ more bone ingrowth inside the implanted material with Ostim ${ }^{\circledR}$ compared to Alpha BSM ${ }^{\circledR}$. BMC Musculoskelet. Disord. 10, 164 (2009).

13. Spies, C., Schnürer, S., Gotterbarm, T. \& Breusch, S. Tierexperimentelle Untersuchung des Knochenersatzstoffs Ostim ${ }^{\mathrm{TM}}$ im knöchernen Lager des Göttinger Miniaturschweins. Z. Für Orthop. Unfallchirurgie 146, 64-69 (2008). 
14. Laschke, M. W., Witt, K., Pohlemann, T. \& Menger, M. D. Injectable nanocrystalline hydroxyapatite paste for bone substitution: In vivo analysis of biocompatibility and vascularization. J. Biomed. Mater. Res. B Appl. Biomater. 82B, 494-505 (2007).

15. Xu, Z., Liu, C., Wei, J. \& Sun, J. Effects of four types of hydroxyapatite nanoparticles with different nanocrystal morphologies and sizes on apoptosis in rat osteoblasts. J. Appl. Toxicol. JAT 32, 429-435 (2012).

16. Qiang Fu et al. In vitro study on different cell response to spherical hydroxyapatite nanoparticles. J. Biomater. Appl. 23, 37-50 (2008).

17. Wu, F. et al. Effect of the materials properties of hydroxyapatite nanoparticles on fibronectin deposition and conformation. Cryst. Growth Des. 15, 2452-2460 (2015).

18. Matsui, N., Nozaki, K., Ishihara, K., Yamashita, K. \& Nagai, A. Concentration-dependent effects of fibronectin adsorbed on hydroxyapatite surfaces on osteoblast adhesion. Mater. Sci. Eng. C Mater. Biol. Appl. 48, 378-383 (2015).

19. Hindié, M. et al. Effects of human fibronectin and human serum albumin sequential adsorption on preosteoblastic cell adhesion. Biointerphases 9, 29008 (2014).

20. Lin, K., Wu, C. \& Chang, J. Advances in synthesis of calcium phosphate crystals with controlled size and shape. Acta Biomater. 10, 4071-4102 (2014).

21. Lin, K. et al. Tailoring the Nanostructured Surfaces of Hydroxyapatite Bioceramics to Promote Protein Adsorption, Osteoblast Growth, and Osteogenic Differentiation. ACS Appl. Mater. Interfaces 5, 8008-8017 (2013).

22. Xia, L. et al. Enhanced osteogenesis through nano-structured surface design of macroporous hydroxyapatite bioceramic scaffolds via activation of ERK and p38 MAPK signaling pathways. J. Mater. Chem. B 1, 5403-5416 (2013).

23. Pepla, E., Besharat, L. K., Palaia, G., Tenore, G. \& Migliau, G. Nano-hydroxyapatite and its applications in preventive, restorative and regenerative dentistry: a review of literature. Ann. Stomatol. (Roma) 5, 108-114 (2014).

24. Nosouhian, S., Razavi, M., Jafari-Pozve, N. \& Rismanchian, M. Comparative evaluation of hydroxyapatite and nano-bioglass in two forms of conventional micro- and nano-particles in repairing bone defects (an animal study). Indian J. Dent. Res. Off. Publ. Indian Soc. Dent. Res. 26, 366-371 (2015).

25. Pinnock, A. et al. Effect of 2D and 3D culture techniques on the in vitro biocompatibility of nanohydroxyapatites. J. Tissue Eng. Regen. Med. 8, 323 (2014).

26. Klesing, J. et al. An injectable paste of calcium phosphate nanorods, functionalized with nucleic acids, for cell transfection and gene silencing. J. Mater. Chem. 20, 6144-6148 (2010).

27. Liao, C. J., Lin, F. H., Chen, K. S. \& Sun, J. S. Thermal decomposition and reconstitution of hydroxyapatite in air atmosphere. Biomaterials 20, 1807-1813 (1999).

28. Viswanath, B. \& Ravishankar, N. Controlled synthesis of plate-shaped hydroxyapatite and implications for the morphology of the apatite phase in bone. Biomaterials 29, 4855-4863 (2008).

29. Willie, B. M., Bloebaum, R. D., Bireley, W. R., Bachus, K. N. \& Hofmann, A. A. Determining relevance of a weight-bearing ovine model for bone ingrowth assessment. J. Biomed. Mater. Res. A 69, 567-576 (2004).

30. Pearce, A. I., Richards, R. G., Milz, S., Schneider, E. \& Pearce, S. G. Animal models for implant biomaterial research in bone: a review. Eur Cell Mater 13, 1-10 (2007).

31. Turner, A. \& Villanueva, A. Static and dynamic histomorphometric data in 9- to 11-year old ewes. ACVS 413 (1993).

32. Canuto, R. A. et al. Hydroxyapatite paste Ostim ${ }^{\circledR}$, without elevation of full-thickness flaps, improves alveolar healing stimulating BMP- and VEGF-mediated signal pathways: an experimental study in humans. Clin. Oral Implants Res. 24, 42-48 (2013).

33. Thorwarth, M., Schultze-Mosgau, S., Kessler, P., Wiltfang, J. \& Schlegel, K. A. Bone Regeneration in Osseous Defects Using a Resorbable Nanoparticular Hydroxyapatite. J. Oral Maxillofac. Surg. 63, 1626-1633 (2005).

34. Zaffe, D., Traversa, G., Mozzati, M., Morelli, F. \& D’Angeli, G. Behavior of aqueous nanocrystalline hydroxyapatite in oral bone regeneration. J. Appl. Biomater. Biomech. 9, 19-25 (2011).

35. Dau, M. et al. Bone formation in mono cortical mandibular critical size defects after augmentation with two synthetic nanostructured and one xenogenous hydroxyapatite bone substitute - in vivo animal study. Clin. Oral Implants Res. 27, 597-603 (2016).

36. Fellah, B. H., Gauthier, O., Weiss, P., Chappard, D. \& Layrolle, P. Osteogenicity of biphasic calcium phosphate ceramics and bone autograft in a goat model. Biomaterials 29, 1177-1188 (2008).

37. Zizelmann, C. et al. Bone formation after sinus augmentation with engineered bone. Clin. Oral Implants Res. 18, 69-73 (2007).

38. van der Meij, A. J. et al. Computed tomography in evaluation of early secondary bone grafting. Int. J. Oral Maxillofac. Surg. 23, 132-136 (1994)

39. Lumetti, S. et al. Correlation between density and resorption of fresh-frozen and autogenous bone grafts. BioMed Res. Int. 2014, 508328 (2014).

40. Ding, T., Xue, Y., Lu, H., Huang, Z. \& Sun, J. Effect of particle size of hydroxyapatite nanoparticles on its biocompatibility. IEEE Trans. Nanobioscience 11, 336-340 (2012).

41. Napierska, D. et al. Size-dependent cytotoxicity of monodisperse silica nanoparticles in human endothelial cells. Small Weinh. Bergstr. Ger. 5, 846-853 (2009).

42. Nabeshi, H. et al. Size-dependent cytotoxic effects of amorphous silica nanoparticles on Langerhans cells. Pharm. 65, 199-201 (2010).

43. Turkez, H. et al. Evaluation of cytotoxic, oxidative stress and genotoxic responses of hydroxyapatite nanoparticles on human blood cells. J. Appl. Toxicol. JAT 34, 373-379 (2014).

44. Al Machot, E., Hoffmann, T., Lorenz, K., Khalili, I. \& Noack, B. Clinical outcomes after treatment of periodontal intrabony defects with nanocrystalline hydroxyapatite (Ostim) or enamel matrix derivatives (Emdogain): a randomized controlled clinical trial. BioMed Res. Int. 2014, 786353 (2014).

45. Chitsazi, M.-T., Shirmohammadi, A., Faramarzie, M., Pourabbas, R. \& Rostamzadeh, A. naser. A clinical comparison of nanocrystalline hydroxyapatite (Ostim) and autogenous bone graft in the treatment of periodontal intrabony defects. Med. Oral Patol. Oral Cir. Bucal 16, e448-453 (2011).

46. Huber, F.-X. et al. [Filling of metaphyseal defects with nanocrystalline hydroxyapatite (Ostim) for fractures of the radius]. Zentralblatt Für Chir. 133, 577-581 (2008).

47. Smeets, R. et al. [Hydroxyapatite bone substitute (Ostim) in sinus floor elevation. Maxillary sinus floor augmentation: bone regeneration by means of a nanocrystalline in-phase hydroxyapatite (Ostim)]. Schweiz. Monatsschrift Für Zahnmed. Rev. Mens. Suisse Odonto-Stomatol. Riv. Mens. Svizzera Odontol. E Stomatol. SSO 118, 203-212 (2008).

48. Huber, F.-X. et al. First histological observations on the incorporation of a novel nanocrystalline hydroxyapatite paste OSTIM in human cancellous bone. BMC Musculoskelet. Disord. 7, 50 (2006).

49. Silva, V. M. T. M., Quadros, P. A., Laranjeira, P. E. M. S. C., Dias, M. M. \& Lopes, J. C. B. A Novel Continuous Industrial Process for Producing Hydroxyapatite Nanoparticles. J. Dispers. Sci. Technol. 29, 542-547 (2008).

50. Brito Lopes, J. C., Miranda Dos Santos Da Costa Laranjeira, P. E., Queiroz Dias, M. M. \& Areosa Martins, A. A. Network Mixer and Related Mixing Process (2005).

51. Knabe, C. et al. Effect of silicon-doped calcium phosphate bone substitutes on bone formation and osteoblastic phenotype expression. Key Eng. Mater. 614, 31-34 (2014).

52. Donath, K. Die Trenn-Dünnschliff-Technik zur Herstellung histologischer Präparate von nicht schneidbaren Geweben und Materialien. Präp. 197-206 (1988). 
53. Hothorn, T., Bretz, F. \& Westfall, P. Simultaneous inference in general parametric models. Biom. J. Biom. Z. 50, 346-363 (2008).

54. R. Core Team. A Language and Environment for Statistical Computing. (R Foundation for Statistical Computing, 2015).

55. Wickham, H. ggplot2: elegant graphics for data analysis. (Springer, 2009).

\section{Acknowledgements}

The authors would like to thank Stefan Lettner, Karl Kropik, Ute Altmann and Antje Genzel for technical assistance and helpful discussion. The research leading to these results has received funding from the European Research Council under the European Community's Seventh Framework Program IMCOSS (FP7-SME- 315679).

\section{Author Contributions}

V.H., S.T., C.M., D.B., H.R. and T.N. conceived and designed the project; V.H., Y.R., C.K., J.F. and T.N. performed experiments; V.H., S.T., Y.R., G.M., P.H. and T.N. analyzed and interpreted the data; R.G., W.A. and P.Q. developed, fabricated and provided the experimental paste materials for the study; V.H., S.T. and Y.R. wrote the manuscript; all authors reviewed the manuscript.

\section{Additional Information}

Supplementary information accompanies this paper at http://www.nature.com/srep

Competing financial interests: The authors declare no competing financial interests.

How to cite this article: Hruschka, V. et al. Comparison of nanoparticular hydroxyapatite pastes of different particle content and size in a novel scapula defect model. Sci. Rep. 7, 43425; doi: 10.1038/srep43425 (2017).

Publisher's note: Springer Nature remains neutral with regard to jurisdictional claims in published maps and institutional affiliations.

(c) (i) This work is licensed under a Creative Commons Attribution 4.0 International License. The images or other third party material in this article are included in the article's Creative Commons license, unless indicated otherwise in the credit line; if the material is not included under the Creative Commons license, users will need to obtain permission from the license holder to reproduce the material. To view a copy of this license, visit http://creativecommons.org/licenses/by/4.0/

(c) The Author(s) 2017 